\title{
Steady wave drift force on basic objects of symmetry
}

\author{
S. Chakrabarti \& A. Gupta \\ University of Illinois at Chicago, Chicago, IL, USA
}

\begin{abstract}
The steady wave drift force on a submerged body is a second-order quantity. With a potential flow assumption, the force arises from the diffraction and radiation of the waves from the interaction with the body. For a fixed body in waves the steady force is contributed from the wave diffraction effect alone. Numerical solutions are generally needed for the computation of the steady drift force on submerged structures. In this paper the steady wave drift forces on several fixed bodies of basic shapes are derived in closed form. The paper addresses the steady drift forces on the following basic structures: a vertical circular cylinder, a submerged horizontal cylinder, a bottom-seated horizontal half cylinder, and a bottom-seated hemisphere. The results developed demonstrate the importance of various independent non-dimensional parameters. A numerical program based on linear diffraction/radiation theory is used to validate the closed form solution.
\end{abstract}

Keywords: basic objects of symmetry, closed form solution, design curves, steady drift force, wave structure interaction.

\section{Introduction}

The linear wave forces on a large structure are computed from the linear diffraction theory based on the (Bernoulli's) linear pressure term in its equilibrium position up to the still water level. However, the structure motion, wave free surface, and the (Bernoulli's) nonlinear pressure terms introduce nonlinear forces on the structure not predictable by the linear theory. The steady wave drift force has been shown to derive from the first order wave potential. The 3-D diffraction/radiation theory is well established to compute these forces. 
For floating offshore structures, the steady drift force can be an important design contribution, especially for soft moored systems.

The purpose of this paper is to derive the steady drift forces on a few fixed bodies of basic shapes in closed form and discuss the contribution of various parameters and different terms in the total drift forces on these fixed bodies. The results presented here for these basic shapes should be useful to designers of offshore structures, which is generally composed of these basic components, in order to evaluate the magnitudes of these forces and to determine the importance of the steady drift force for these components.

\section{Literature review}

Much work has already been done with the numerical computation of steady drift forces. These works have been carried out assuming the structure to be rigid and either freely floating or fixed. They are too many to cite here. One noteworthy one is by Pinkster [5] who studied second order steady drift forces and low frequency forces using 3-D diffraction/radiation theory. Ogilvie [6] summarized both momentum and pressure integration approach for drift force calculations.

Skourup et al. [7] investigated non-linear loads on fixed body due to waves and current using potential theory and 3-D Boundary Element Method and computed second order steady and oscillatory forces on a vertical circular cylinder.

\section{Theoretical background}

The linear diffraction theory is based on a velocity potential function $\Phi$ which is composed of an incident wave potential, and a scattered potential from the fixed body. Once the velocity potential is known the steady drift forces on the fixed body are computed from two sources.

If the body extends above the mean water level, the steady drift force due to free surface effect is

$$
F_{21 i}=\frac{1}{2} \rho g \int_{W L} \eta_{r}^{2} n_{i} d l
$$

where $\rho=$ water mass density, $\mathrm{g}=$ gravitational acceleration, $\eta_{r}=$ wave elevation at the body surface including diffraction effect, $i=$ direction of the drift force component, $n_{i}=$ surface direction normal component along $i$, and $\mathrm{WL}=$ water line of the body in its equilibrium position. The second component is the steady drift force due to Bernoulli's velocity-squared term

$$
F_{22 i}=\frac{1}{2} \iint_{S_{0}} \rho \sum_{j=1}^{3} u_{j}^{2} n_{i} d S
$$

where $\mathrm{u}_{j}$ represents the water particle velocity components along the three body coordinates based on the first-order velocity potential and $d S$ is the elemental area on the submerged part of the body surface up to the still water level, $S_{0}$. 


\section{Closed form formulas}

The steady drift force due to the incident wave on any symmetric basic shape (with respect to the wave direction) can be shown to be identically zero due the symmetry of the pressure distribution. Thus the contribution to the second-order drift force comes from the diffraction potential.

\subsection{Vertical cylinder}

Let us consider a vertical cylinder resting on the ocean floor and extending above the free surface. Vertical cylinder is the most researched shape for wave forces. It has led to many studies for decades (analytical, numerical and experimental), and linear results, second-order results and fully non-linear results for the wave forces and the wave runup on the cylinder can be found in the literature. For a bottom-seated cylinder extended above the free surface, MacCamy and Fuchs [4] developed the forces on a fixed vertical cylinder in a closed form using the Bessel function series. The closed form expression for the first-order velocity potential was used by several researchers to derive the second order components of the forces (see, e.g.. Chakrabarti [1]).

While it is one of the simplest shapes, it has several offshore applications including the deep draft floating SPAR in deep water in waves whose pressure decays to near-zero at the bottom of the SPAR.

The total linear velocity potential including diffraction at the surface of a bottom-mounted cylinder, $r=a$ (e.g., Chakrabarti [3]) is written as

$$
\Phi(a, \theta ; t)=\frac{H g}{2 \omega} \frac{2}{\pi k a} \sum_{n=0}^{\infty} \delta_{n}\left[a_{n} \cos \omega t+b_{n} \sin \omega t\right] \cos n \theta
$$

where $a=$ cylinder radius, $H=$ wave height, $\omega=$ wave frequency, $k=$ wave number, $t=$ time, $(r, \theta)=$ cylindrical polar coordinates, and the coefficients

$$
\begin{gathered}
a_{2 n}=(-1)^{n} \frac{J_{2 n}^{\prime}(k a)}{A_{2 n}(k a)} ; a_{2 n+1}=(-1)^{n+1} \frac{Y_{2 n+1}^{\prime}(k a)}{A_{2 n+1}(k a)} ; b_{2 n}=-(-1)^{n} \frac{Y_{2 n}^{\prime}(k a)}{A_{2 n}(k a)} ; \\
b_{2 n+1}=(-1)^{n} \frac{J_{2 n+1}^{\prime}(k a)}{A_{2 n+1}(k a)} ; \text { and } A_{n}=\left[J_{n}^{\prime}(k a)\right]^{2}+\left[Y_{n}^{\prime}(k a)\right]^{2}
\end{gathered}
$$

and the functions $J^{\prime}(\mathrm{ka})$ and $Y^{\prime}(\mathrm{ka})$ are the derivatives of the Bessel function of the first and second kind. The first-order wave profile at the surface of the cylinder, $r=a$, becomes:

$$
\eta(a, \theta ; t)=\frac{2}{\pi k a} \frac{H}{2} \sum_{n=0}^{\infty} \delta_{n}\left[-a_{n} \sin \omega t+b_{n} \cos \omega t\right] \cos n \theta
$$


Upon integration of Eq. (1) around the cylinder the non-dimensional component of the horizontal steady drift force due to the free surface at the vertical cylinder reduces to

$$
\frac{\bar{F}_{21 x}}{\rho g(H / 2)^{2} a}=\frac{4}{\pi(k a)^{2}} \sum_{n=0}^{\infty}\left(a_{n} a_{n+1}+b_{n} b_{n+1}\right)
$$

Substituting

$$
a_{n} a_{n+1}+b_{n} b_{n+1}=\frac{2}{\pi k a}\left(1-\frac{n(n+1)}{(k a)^{2}}\right) \frac{1}{A_{n} A_{n+1}}
$$

the above expression reduces to

$$
\frac{\bar{F}_{21 x}}{\rho g(H / 2)^{2} a}=\frac{8}{\pi^{2}(k a)^{3}} \sum_{n=0}^{\infty}\left(1-\frac{n(n+1)}{(k a)^{2}}\right) \frac{1}{A_{n} A_{n+1}}
$$

which is a function of $k a$ only and does not depend on the water depth. The nondimensional mean velocity-squared force has the form:

$$
\frac{\bar{F}_{22 x}}{\rho g(H / 2)^{2} a}=\frac{4}{\pi^{2}(k a)^{3}}\left\{\begin{array}{l}
-\left[1-\frac{2 k D}{\sinh 2 k D}\right] \sum_{n=0}^{\infty}\left(1-\frac{n(n+1)}{(k a)^{2}}\right) \frac{1}{A_{n} A_{n+1}}+ \\
{\left[1+\frac{2 k D}{\sinh 2 k D}\right] \frac{1}{(k a)^{2}} \sum_{n=0}^{\infty} n(n+1)\left(1-\frac{n(n+1)}{(k a)^{2}}\right) \frac{1}{A_{n} A_{n+1}}}
\end{array}\right\}
$$

In deep water combining the two expressions, the total drift force on the vertical cylinder is

$$
\frac{\bar{F}_{2 x}}{\rho g(H / 2)^{2} a}=\frac{4}{\pi^{2}(k a)^{3}} \sum_{n=0}^{\infty}\left[1+\left(\frac{n(n+1)}{(k a)^{2}}\right)\right]^{2} \frac{1}{A_{n} A_{n+1}}
$$

The non-dimensional steady forces (Eqs. (7)-(8)) on a bottom-seated vertical cylinder for various values of $d / a$ are given in Fig. 1 as functions of $k a$. The free surface contribution is independent of the water depth and is positive. The velocity-squared term is negative and is only a weak function of water depth at the low values of $k a$ (long periods). The net steady drift force on the cylinder is positive and increases with the higher ka values (short periods). It is also relatively independent of the water depth. The closed form solution is validated in Fig. 2 by comparing the results with the numerical solution based on the well-established boundary element linear diffraction theory. The cylinder in the numerical solution was represented by 2000 panels. Note that the results from Skourup et al. [7] also match well (Fig. 2). 


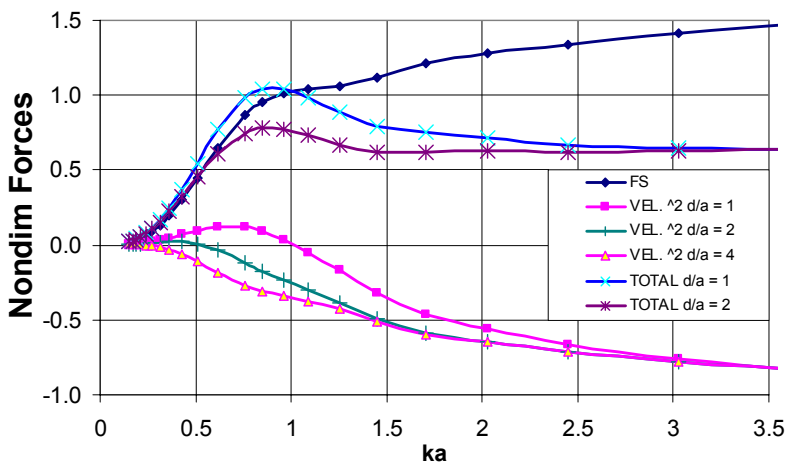

Figure 1: Horizontal steady drift force on a vertical cylinder.
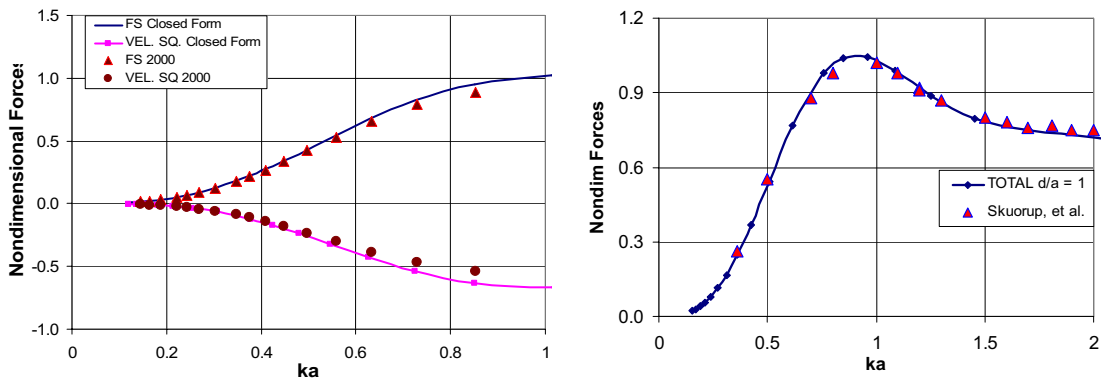

Figure 2: $\quad$ Validation of steady drift force on a vertical cylinder.

\subsection{Bottom-mounted horizontal half-cylinder}

Let us now consider a horizontal half-cylinder seated on the ocean bottom (Chakrabarti [2]). A possible application of this geometry is the buried ocean floor pipelines used for the transportation of crude oil from the production platform to shuttle tankers or shore.

An appropriate cylindrical coordinate system $(r, \theta)$ is chosen to describe the velocity potential. Based on the linear diffraction theory for a bottom-seated halfcylinder the velocity potential at the surface of the cylinder, $r=a$, can be expressed in a closed form as long as the half cylinder is removed from the free surface so that the effects of the free surface on the velocity potential is considered small and can be ignored. In this case the velocity potential becomes

$$
\Phi(r, \theta ; t)=i \frac{g H}{2 \omega} \frac{1}{\cosh k d}\left[\begin{array}{l}
\cosh (k r \sin \theta) \exp (i k r \cos \theta)+ \\
\left.\cosh \left[k\left(\frac{a^{2}}{r}\right) \sin \theta\right] \exp \left[i k\left(\frac{a^{2}}{r}\right) \cos \theta\right]-1\right] \exp (-i \omega t)
\end{array}\right.
$$


For the half-cylinder on the ocean bottom it has been shown that an accurate first order solution can be obtained as long as $d / a$ is equal to 2 or greater.

The steady horizontal drift force for a submerged half-cylinder due to the velocity-squared pressures is identically zero. The vertical component of the drift force is obtained from the integral

$$
F_{2 y}=L \int_{0}^{\pi} p \sin \theta(a d \theta)
$$

where the dynamic pressure is derived from the velocity-squared term. Since the normal velocity on the surface of the cylinder is zero, the only contribution to the velocity-squared term on the 2-D half-cylinder is

$$
p=\frac{1}{2} \rho u_{\theta}^{2}=\frac{1}{2} \rho\left(\frac{1}{r} \frac{\partial \Phi}{\partial \theta}\right)^{2}
$$

Then

$$
\bar{F}_{2 y}=\frac{F_{2 y}}{\rho g L(H / 2)^{2}}=\left(\frac{2 k a}{\sinh 2 k d}\right) \int_{0}^{\pi}\left[\sinh ^{2}(k a \sin \theta)-\cos ^{2} \theta\right] \sin \theta d \theta
$$

which reduces to

$$
\bar{F}_{2 y}=\left(\frac{2 k a}{\sinh 2 k d}\right)\left[I_{1}(k a)+\frac{1}{3}\right] ; \quad I_{1}(k a)=\int_{0}^{\pi} \cosh (2 k a \sin \theta) \sin \theta d \theta
$$

The values of the integral $I_{1}(k a)$ versus $k a$ are given in Table 1 .
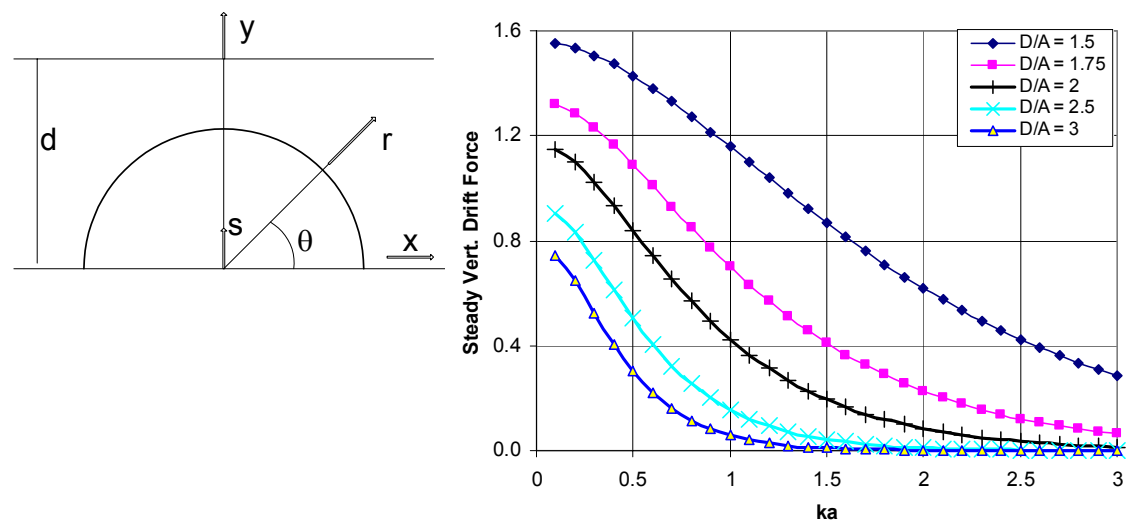

Figure 3: $\quad$ Vertical steady drift force on a horizontal half-cylinder. 
The results on the steady drift force versus $k a$ are given in Fig. 3 for various depths of submergence. For brevity the results are shown for $d / a$ value down to 1.5. It can be seen that the forces increase in value as the $k a$ value decreases. As expected, the magnitude of the non-dimensional force decreases rapidly with increase in the water depth.

\subsection{Deep-submerged horizontal cylinder}

For a deeply submerged horizontal cylinder away from the free surface the effect of the free surface may be ignored. It is also assumed that the cylinder is somewhat removed from the ocean floor. In this case, a closed form expression of the velocity potential has the form quite similar to the velocity potential for the half-cylinder, Eq. (10), by introducing the depth of submergence $k s_{0}$ in the cosh terms. By the same reasoning the validity of this potential function for the first-order force can be shown to be applicable for a $d / a$ value of at least equal to 2. A possible application of this geometry is a submerged component of an offshore structure.

The time independent velocity-squared pressure is obtained from the velocity potential as

$$
p=\frac{\rho g}{a}\left(\frac{H}{2}\right)^{2}\left(\frac{2 k a}{\sinh 2 k d}\right)\left[\begin{array}{l}
\cos ^{2} \theta \sinh ^{2}\left(k a \sin \theta+k s_{0}\right) \\
+\sin ^{2} \theta \cosh ^{2}\left(k a \sin \theta+k s_{0}\right)
\end{array}\right]
$$

The horizontal component of the force is identically zero. The vertical component is given by

$$
F_{2 y}=L \int_{0}^{\pi} p \sin \theta(a d \theta)
$$

Then

$$
\frac{F_{2 y}}{\rho g L(H / 2)^{2}}=\left(\frac{2 k a}{\sinh 2 k d}\right)_{0}^{\pi}\left[\begin{array}{l}
\cos ^{2} \theta \sinh ^{2}\left(k a \sin \theta+k s_{0}\right) \\
+\sin ^{2} \theta \cosh ^{2}\left(k a \sin \theta+k s_{0}\right)
\end{array}\right] \sin \theta d \theta
$$

which reduces to

$$
\bar{F}_{2 y}=\left(\frac{2 k a}{\sinh 2 k d}\right)\left[I_{2}\left(k a, s_{0} / a\right)+\frac{1}{3}\right] ; \quad I_{2}\left(k a, s_{0} / a\right)=\int_{0}^{\pi} \cosh \left(2\left(k a\left(\sin \theta+s_{0} / a\right)\right)\right) \sin \theta d \theta
$$

Table 1 lists the values of $I_{2}\left(k a, s_{0} / a\right)$ for different values of $s_{0} / a$. The results on the steady drift force versus $k a$ are given in Fig. 4 for various depths of submergence $s_{0} / a$. It can be seen that the forces peak at intermediate $k a$ values. As expected, the magnitude of the non-dimensional force decreases rapidly with the depth of submergence as well as $k a$ values. 
Table 1: $\quad$ Values of integrals $I_{1}(k a)$ and $I_{2}\left(k a, s_{0} / a\right)$.

\begin{tabular}{|l|l|l|l|l|l|}
\hline$k a$ & $I_{1}(k a)$ & $I_{2}\left(k a, s_{0} / a\right)$ & $I_{2}\left(k a, s_{0} / a\right)$ & $I_{2}\left(k a, s_{0} / a\right)$ & $I_{2}\left(k a, s_{0} / a\right)$ \\
\hline & & $s / a=1$ & $s / \mathrm{a}=1.5$ & $s / a=2$ & $s / a=2.5$ \\
\hline 0.1 & 2.0267 & 0.021637 & 0.032725 & 0.044142 & 0.056 \\
\hline 0.2 & 2.1078 & 0.058073 & 0.090012 & 0.12556 & 0.16615 \\
\hline 0.3 & 2.2458 & 0.075159 & 0.12118 & 0.1782 & 0.25137 \\
\hline 0.4 & 2.4452 & 0.070808 & 0.12035 & 0.1894 & 0.28917 \\
\hline 0.5 & 2.7124 & 0.056239 & 0.1019 & 0.17356 & 0.28953 \\
\hline 0.6 & 3.056 & 0.040324 & 0.078597 & 0.14602 & 0.26762 \\
\hline 0.7 & 3.4872 & 0.02707 & 0.057172 & 0.11645 & 0.23516 \\
\hline 0.8 & 4.0201 & 0.017382 & 0.039996 & 0.089602 & 0.19968 \\
\hline 0.9 & 4.672 & 0.010818 & 0.027232 & 0.067232 & 0.16547 \\
\hline 1 & 5.4644 & 0.0065826 & 0.018182 & 0.04953 & 0.13468 \\
\hline 1.1 & 6.4233 & 0.0039392 & 0.011965 & 0.035988 & 0.10813 \\
\hline 1.2 & 7.5807 & 0.0023278 & 0.0077869 & 0.025871 & 0.0859 \\
\hline 1.3 & 8.975 & 0.0013623 & 0.0050243 & 0.018443 & 0.067674 \\
\hline 1.4 & 10.653 & 0.0007912 & 0.0032196 & 0.013059 & 0.052958 \\
\hline 1.5 & 12.671 & 0.00045673 & 0.0020517 & 0.0091964 & 0.041215 \\
\hline 1.6 & 15.097 & 0.00026236 & 0.0013015 & 0.0064469 & 0.031932 \\
\hline 1.7 & 18.013 & 0.00015009 & 0.00082249 & 0.0045024 & 0.024646 \\
\hline 1.8 & 21.519 & $8.5578 \mathrm{e}-05$ & 0.00051809 & 0.0031343 & 0.018962 \\
\hline 1.9 & 25.735 & $4.8654 \mathrm{e}-05$ & 0.00032545 & 0.002176 & 0.014548 \\
\hline 2 & 30.806 & $2.7594 \mathrm{e}-05$ & 0.00020396 & 0.0015071 & 0.011136 \\
\hline 2.1 & 36.906 & $1.5616 \mathrm{e} 005$ & 0.00012755 & 0.0010416 & 0.0085061 \\
\hline 2.2 & 44.248 & $8.8215 \mathrm{e}-06$ & $7.9626 \mathrm{e}-05$ & 0.00071863 & 0.0064856 \\
\hline 2.3 & 53.085 & $4.9751 \mathrm{e}-06$ & $4.9627 \mathrm{e}-05$ & 0.00049499 & 0.0049371 \\
\hline 2.4 & 63.726 & $2.8017 \mathrm{e}-06$ & $3.0886 \mathrm{e}-05$ & 0.00034046 & 0.003753 \\
\hline 2.5 & 76.544 & $1.5757 \mathrm{e}-06$ & $1.9197 \mathrm{e}-05$ & 0.00023387 & 0.0028491 \\
\hline 2.6 & 91.989 & $8.8519 \mathrm{e}-07$ & $1.1918 \mathrm{e}-05$ & 0.00016046 & 0.0021605 \\
\hline 2.7 & 110.61 & $4.9674 \mathrm{e}-07$ & $7.3914 \mathrm{e}-06$ & 0.00010998 & 0.0016365 \\
\hline 2.8 & 133.05 & $2.7848 \mathrm{e}-07$ & $4.5796 \mathrm{e}-06$ & $7.531 \mathrm{e}-05$ & 0.0012384 \\
\hline 2.9 & 160.12 & $1.5599 \mathrm{e}-07$ & $2.8349 \mathrm{e}-06$ & $5.1522 \mathrm{e}-05$ & 0.00093637 \\
\hline 3 & 192.77 & $8.7301 \mathrm{e}-08$ & $1.7535 \mathrm{e}-06$ & $3.522 \mathrm{e}-05$ & 0.00070741 \\
\hline & & & & & \\
\hline
\end{tabular}



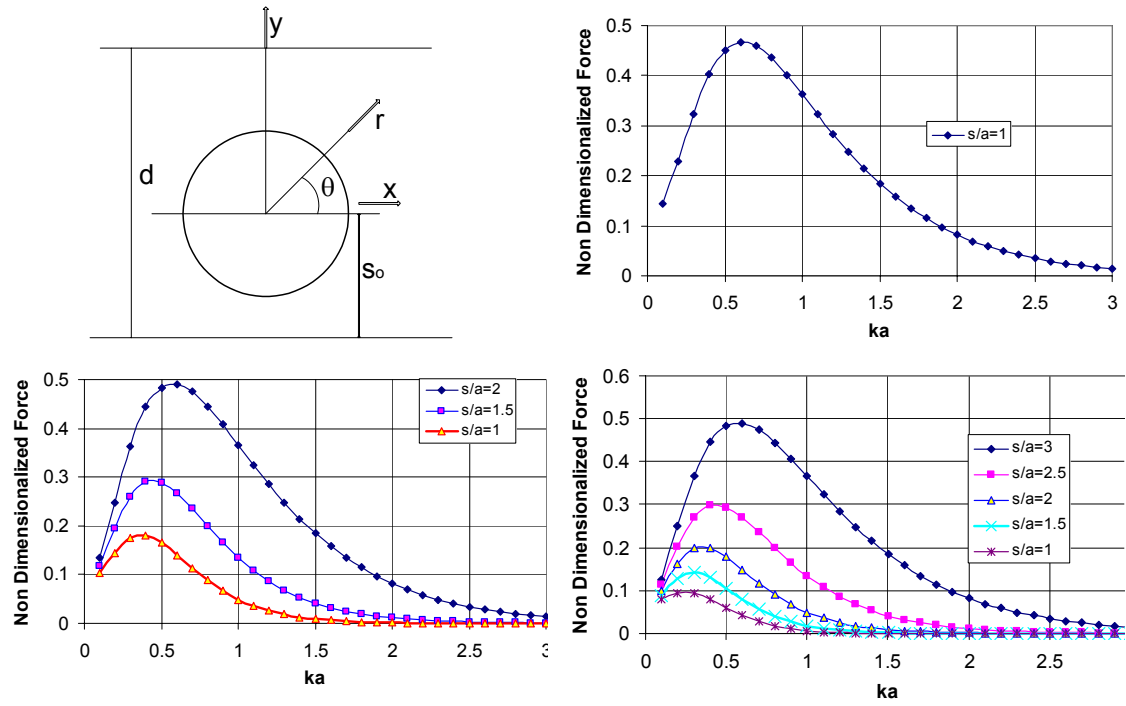

Figure 4: $\quad$ Vertical steady drift force on a horizontal cylinder.

\subsection{Bottom-mounted hemisphere}

Let us consider a hemisphere seated on the ocean bottom (Chakrabarti and Naftzger [2]). The spherical coordinate system is adopted in terms of $\theta$ and $\mu$. Based on the linear diffraction theory for a bottom-seated hemisphere the velocity potential at the surface of the cylinder, $r=a$, can be expressed in a closed form as long as the hemisphere is removed from the free surface with a $d / a$ ratio of at least 1.5 , so that its effects on the velocity potential can be ignored. In this case the velocity potential becomes

$$
\Phi(r, \theta ; t)=i \frac{g H}{2 \omega} \frac{1}{\cosh k d}\left[\begin{array}{l}
\cosh \left(\frac{r}{a} Y\right) \exp \left(i \frac{r}{a} X\right)+\left[\frac{a}{r}+\frac{i X}{R^{2}}\right] \cosh \left(\frac{a}{r} Y\right) \exp \left(i \frac{a}{r} X\right) \\
-\frac{Y}{R^{2}} \sinh \left(\frac{a}{r} Y\right) \exp \left(i \frac{a}{r} X\right)-\frac{i X}{R^{2}}
\end{array}\right] \exp (-i \omega t)
$$

where

$$
X=k a \cos \theta \sin \mu \quad Y=k a \sin \theta \sin \mu \quad R=k a \sin \mu \quad \lambda=g H /(2 \omega \cosh k d)
$$

Then the $\theta$ - and $\mu$-components of the particle velocity respectively are

$$
u_{\theta}(r, \theta ; t)=\frac{1}{a \sin \mu} \frac{\partial \Phi}{\partial \theta}=\frac{\lambda}{a \sin \mu}\left\{T_{1} \sinh Y \cos (X-\omega t)+T_{2} \cosh Y \sin (X-\omega t)-\frac{X}{R^{2}} \sin \omega t\right\}
$$

and

$$
u_{\mu}(r, \theta ; t)=\frac{\cot \mu}{a} \frac{\partial \Phi}{\partial \theta}=\frac{\lambda \cot \mu}{a}\left\{-T_{1} \cosh Y \sin (X-\omega t)+T_{2} \sinh Y \cos (X-\omega t)-\frac{X}{R^{2}} \sin \omega t\right\}
$$


where $T_{1}=2 X-2 R+X / R^{2}$ and $T_{2}=2 Y-Y / R^{2}$. For a bottom-seated hemisphere the steady horizontal surge drift force due to velocity-squared pressures is written as

$$
F_{2 x}=-\frac{1}{2} \rho a^{2} \int_{0}^{\pi}\left(\int_{0}^{\pi}\left(u_{\theta}^{2}+u_{\mu}^{2}\right) \cos \theta d \theta\right) \sin ^{2} \mu d \mu
$$

Substituting the value of $\lambda$ the normalized force reduces to

$$
\left.\left.\frac{F_{2 x}}{\rho g(H / 2)^{2} a}=-\left(\frac{1}{4 k a \sinh 2 k d}\right)_{0}^{\pi} \int_{0}^{\pi}\left\{\begin{array}{l}
\left\{\begin{array}{l}
T_{1}^{2} \sinh ^{2} Y+T_{2}^{2} \cosh ^{2} Y+\left(\frac{Y}{R^{2}}\right)^{2} \\
-2 \frac{Y}{R^{2}} T_{1} \sinh Y \sin X+2 \frac{Y}{R^{2}} T_{2}^{2} \cosh Y \cos X
\end{array}\right\}+ \\
\int_{1}^{2} \cosh ^{2} Y+T_{2}^{2} \sinh ^{2} Y+\left(\frac{X}{R^{2}}\right)^{2} \\
-2 \frac{X}{R^{2}} T_{1} \cosh Y \cos X+2 \frac{X}{R^{2}} T_{2}^{2} \sinh Y \sin X
\end{array}\right\} \cos ^{2} \mu\right) \cos \theta d \theta\right) d \mu
$$

The vertical component of steady drift force is computed from

$$
F_{2 y}=-\frac{1}{2} \rho a^{2} \int_{0}^{\pi}\left(\int_{0}^{\pi}\left(u_{\theta}^{2}+u_{\mu}^{2}\right) \sin \theta d \theta\right) \sin ^{2} \mu d \mu
$$

so that the only change in Eq. 23 is to replace $\cos \theta$ with $\sin \theta$.

The results on the non-dimensional steady horizontal and vertical drift forces versus ka are given in Fig. 5. It can be seen that the forces peak at an intermediate ka value. As expected, the magnitude of the non-dimensional force decreases rapidly with the depth f submergence.
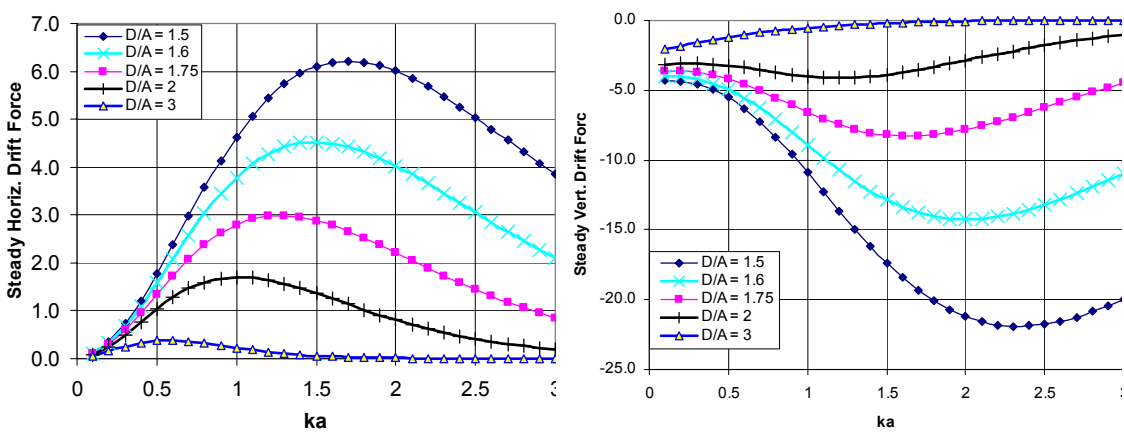

Figure 5: Horizontal and vertical steady drift forces on a bottom-seated hemisphere.

\section{Conclusions}

The second-order steady wave drift forces on a few fixed basic objects of symmetry have been computed based on the total diffraction potential. The 
objects chosen are the vertical cylinder, submerged horizontal cylinder, bottomseated horizontal half-cylinder, and bottom-seated hemisphere. For the vertical cylinder extending above the still water level, the horizontal drift forces receive contributions from the free surface as well as the Bernoulli's velocity-squared terms. For the other fixed bodies the contribution comes from the velocitysquared term only, as the objects do not penetrate the mean water level.

The expressions for the steady forces are obtained in closed forms. It is shown that the forces depend on the non-dimensional diffraction parameter $k a$ and the depth to radius ratio $d / a$. For the submerged horizontal cylinder, the forces are also a function of the non-dimensional submergence depth, $s_{0} / a$.

Comprehensive results are presented for these shapes for different values of these non-dimensional parameters so that they can be used directly to estimate the drift force in a design of these components.

While the results are limited to bodies of specific basic shapes, they constitute members of most offshore structures, including submerged pipelines, caissons, TLP, Spar, and semisubmersible. It is expected that the formulas and numerical results presented here will have applications in the design of such structures.

\section{References}

[1] Chakrabarti, S.K., Nonlinear Wave Forces on Vertical Cylinder, Journal of Hydraulics Division, ASCE, Vol. 98, November 1972.

[2] Chakrabarti, S.K. and Naftzger, R.A., Nonlinear Wave Forces on HalfCylinder and Hemisphere, Journal of Waterways, Harbors and Coastal Engineering Division, ASCE, Vol. 100, WW3, August 1974.

[3] Chakrabarti, S.K., Hydrodynamics of Offshore Structures, WIT Press, Ashurst, Southampton, 1990.

[4] MacCamy, R. C., and Fuchs, R. A., Wave Forces on a Pile: A Diffraction Theory, Technical Memorandum No.69, Beach Erosion Board, 17 pp, 1954.

[5] Pinkster, J.A., Mean and Low Frequency Wave Drifting Forces on Floating Structures, Ocean Engineering, Vol. 6, pp. 593-615, 1979.

[6] Ogilvie, T. F., Second-Order Hydrodynamics Effects on Ocean Platforms, Proceedings International Workshop on Ship and Platform Motions, Berkeley, University of California, pp. 205-265, 1983.

[7] Jesper Skourup, Cheung Kwok Fai, Harry B. Bingham and Bjarne Buchmann, Loads on a 3D body due to Second Order Waves and a Current, Ocean Engineering, Vol. 27 (2000) 707-727. 